\title{
Unextended Complexes
}

\author{
Martin Pickup, New College, Oxford
}

Extended simples are fruitfully discussed in metaphysics. They are entities which are located in a complex region of space but do not themselves have parts. In this paper I will discuss unextended complexes, entities which are not located at a complex region of space but do themselves have parts. In particular, I focus on one type of unextended complex: pointy complexes (entities that have parts but are located at a single point of space). Four areas are indicated where pointy complexes might prove philosophically useful. Unextended complexes are therefore philosophically fruitful, in much the same way as extended simples.

Keywords: Metaphysics, Extended Simples, Mereology, Location, Space, Gunk, Material Objects

There is an interesting and growing literature in contemporary analytic metaphysics considering extended simples. ${ }^{1}$ Extended simples are entities that are extended in space but have no (proper) parts. The debate has focused on three things: (i) whether such entities are (metaphysically) possible, (ii) if so what they would be like, and (iii) what impact their possibility or impossibility might have on other questions in metaphysics. Of course, such entities would be very odd. They would occupy a complex region of space (or spacetime: I'll ignore this distinction from hereon) without

\footnotetext{
${ }^{1}$ As a small sample, see Braddon-Mitchell and Miller (2006), Markosian (2004), McDaniel (2007a \& 2009), Parsons (2000 \& 2007) and Simons (2004).
} 
themselves being complex. Thus the mereological structure of the entity and the mereological structure of its location would not align.

We take it for granted that, typically, the mereological structure of object and their locations are in fact aligned (Schaffer (2009) calls this 'mereological harmony'). If the structure of entities and their locations must be aligned, then extended simples are metaphysically impossible. But why must they be aligned? ${ }^{2}$ Although extended simples would certainly be odd, it isn't clear they are impossible. Extended simples shouldn't be ruled out without good reason: they remain epistemically possible. In this paper I would like to highlight another type of entity for similar consideration: unextended complexes. Unextended complexes are entities that are not extended in space but have (proper) parts. What I call 'unextended complexes' have been introduced to the literature in a few different places. ${ }^{3}$ But they have so far been primarily discussed briefly, parenthetically and instrumentally, as a means to defend or attack some other claims. It also seems worthwhile to consider them as entities of philosophical interest in their own right. In this short paper, I will not be able to do so in full, but I hope to flag some interesting avenues of investigation that unextended complexes open up.

\footnotetext{
${ }^{2}$ One reason would be if entities are somehow composed of the regions they occupy (Schaffer 2009). Other reasons can be given. But none will be indisputable. See Saucedo (2011) for a detailed and interesting discussion of ways that the structure of space and the mereological structure of objects might come apart. ${ }^{3}$ There are two main areas in which unextended complexes have been discussed. Armstrong seems to endorse such entities, in the context of providing universals for quantities. He offers the suggestion that point particles have proper parts. In doing so, he makes it possible for these parts can be the bearers of certain quantitative universals that thereby solve an internal problem for his view. See Armstrong (1988) p315 and (1997) p64-65. See also Eddon (2007) Sect. III for a criticism.

The second area is in the literature on mereology and location. Unextended complexes are briefly mentioned in a footnote of Markosian's (Markosian (1998), fn. 20), and Sider is credited with raising them. Wasserman (2003), McDaniel $(2006,2007 \mathrm{~b})$ and Saucedo (2011) discuss them in various degrees of detail in the context of the relationship between the locations of objects and their mereology. McDaniel (2007b) argues directly for their possibility; in the other papers they are addressed more indirectly. In each case, unextended complexes are pointed out as possible counterexamples to claims about the alignment of the structures of objects and their locations. In particular, they are used to attack principles stating what it takes to be a mereological simple and related claims about the possibility of gunk (see Markosian (1998) and Hudson (2001) for the views against which unextended complexes are here deployed).

Note that all the previous discussion in the literature concerns a particular sort of unextended complex, what I call in the next section 'pointy complexes'.
} 
A good starting point are the parallels of the three questions that have been the focus of the extended simples debate. We can ask (i) whether unextended complexes are (metaphysically) possible, (ii) if they are possible what they would be like, and (iii) what impact their possibility or impossibility might have on other questions in metaphysics. At the very least, considering these questions for unextended complexes might shed light on the more established issues concerning extended simples.

\section{Two types of unextended complexes: abstracta and pointy complexes}

There are two ways for an entity to be an unextended complex, corresponding to two distinct ways to be unextended. Something can be unextended (a) by having no location in space at all or (b) by being located at a simple part of space.

The existence of entities that have no spatial location at all is itself controversial: it is the question of whether there are abstract objects (on one understanding of 'abstract'). So it's disputed whether anything satisfies (a). But if abstract objects are metaphysically possible, then there's an obvious sense in which unextended complexes are plausibly metaphysically possible. If any abstract object has (proper) parts, then we have an unextended object which is complex. ${ }^{4}$ In other words, if it's metaphysically possible for there to be a complex abstract object, it's metaphysically possible for there to be an unextended complex. Examples that might be given include complex universals (the property of being 'red and heavy', for instance), sets that are not singletons (the set of all things in this room, for instance), and complex propositions (the proposition ' $p$ or $q$ ', for instance).

This is already an interesting result. For it puts pressure on the claim that the mereological structure of entities and their locations must align of metaphysical necessity: if we accept complex abstract objects we seem to have an immediate counterexample. Thus the possibility of abstract complexes

\footnotetext{
${ }^{4} \mathrm{~A}$ contrarian might argue that even if there are abstract objects, they can't be complex. This is a reasonable move, but we need to hear the argument.
} 
would undermine an argument against the possibility of extended simples that used such a principle.

(Of course, the critic of extended simples could recover a restricted version of the principle that claims that if they have a location, the mereological structure of entities and their locations necessarily align. This would still tell against extended simples.)

But many deny that there are abstract objects. Those who deny abstract objects are also likely to deny that abstract objects are metaphysically possible. ${ }^{5}$ So they will not accept the above argument for the metaphysical possibility of unextended complexes, and against mereological harmony. However, I think the weight of the majority would favour the claim that abstract object with proper parts are at least epistemically possible, i.e. we shouldn't rule out complex abstract objects. If this is so, then we also shouldn't rule out unextended complexes. This is a weaker claim than the claim that unextended complexes are metaphysically possible, but it further underwrites their interest. At the very least, we stand in need of a reason to reject complex abstract objects. ${ }^{6}$

However, I think abstract complexes, while they are indeed unextended complexes, are less interesting than entities that would be unextended complexes in virtue of satisfying (b) above: namely entities that are complex but are located in a simple place. The obvious way for an entity to be located at a simple place is for it to be located at exactly one point of space. ${ }^{7}$ An entity of this sort

\footnotetext{
${ }^{5}$ There is space for a view whereby there are in fact no abstract objects, but they remain metaphysically possible. Those subscribing to such a view would be sympathetic to my point above. Thanks to a reviewer for prompting me to revise this section.

${ }^{6}$ If an argument can be given for the metaphysical necessity of a mereological harmony principle, then this would be such a reason.

${ }^{7}$ A reviewer has pointed out an alternative way for an entity to occupy a simple place: for the location of the entity to be an extended simple region of space. In other words, an entity might be an unextended complex by being an entity with mereological structure but located in a region of space that itself lacks mereological structure and is hence simple (rather than at a point of space). In order to make sense of such a possibility, being an extended region must not entail being a complex region. Given that one natural way to understand what it is to be an extended region is as being composed of more than one point, an alternative account of extension for regions would be needed.

I think this is an interesting idea. I believe that what I say about pointy complexes could be generalised to include such cases. (A possible exception is the discussion in Sect. 2.1, where what account we give of extended regions could matter for how things could be located at them.) I won't develop the generalised argument in what follows in order to focus on pointy complexes, which are my main target.
} 
will be unextended by being located at a single point of space. I will call such entities pointy complexes.

A pointy complex would be an entity that has a spatial location, but that location is simple. The entity itself, by contrast, has proper parts. Just as in the case of an extended simple the mereological structure of the place the entity is located outruns the mereological structure of the entity, so in the case of a pointy complex the mereological structure of the entity outruns the mereological structure of the place it is located.

This does seem strange. How could it be that something located at a single point of space has proper parts? But I contend that it is no stranger than the extended simple case, where something located at more than one point of space has no proper parts (I'll come back to this in 2.4). More provisionally: until a reason is given why pointy complexes are worse off than extended simples, we should treat their possibility equally. ${ }^{8}$

In the next section I want to briefly indicate some non-exhaustive areas where pointy complexes can be fruitfully considered.

\section{Some applications of pointy complexes}

In general, unextended complexes (and in particular pointy complexes) are an additional fruitful resource for examining the issues in metaphysics where extended simples have been deployed. Unextended complex will also, I hope, give us an interesting perspective on what extended simples themselves are and their role. Here l'll briefly outline four ways that pointy complexes can

\footnotetext{
${ }^{8}$ One such reason might be offered: there are no such thing as spatial (or spacetime) points. Although this would rule out pointy complexes, it wouldn't rule out a more general case where entities have a mereological structure which outruns the structure of their locations. Most of what I wish to do with pointy complexes can be done with entities whose mereological structure is simply more complex than their location structure. It is the lack of isomorphism between these that is the central dynamic. (Note that the same generalisation applies to extended simples for those who think there are no mereological atoms.) See the previous footnote for an alternative that permits simple places without points of space. Also see McDaniel (2007b), p239-241 for an argument for the metaphysical possibility of unextended complexes.
} 
supplement some relevant debates. Note that although parallels are drawn between the roles of extended simples and unextended complexes, the content of the deployment of each is distinct. Although I am taking unextended complexes to offer structurally similar moves to those made using extended simples, the actual results of these moves are quite different.

(i) One way extended simples have been used is to put pressure on our normal notions concerning entities' location. For what is the location of the extended simple? There are two main options for answering this question. The first is to take extended simples as multiply located. That is, the extended simple is fully located many times over: it occupies each of the points that make up its extension. The extended simple is akin to a time-travelling enduring object that thereby gains additional locations in its own past. The second option is to take extended simples as spanners. That is, the extended simple is fully located at only one place: the fusion of all the points that make up its extension. It spans this region without being located at any part of it. Either way, multilocation and spanning both give us novel ways to think about location concepts: either entities can be exactly located at more than once over or entities can have locations without being located at any subregions of that location.

In a parallel way, pointy complexes put us under some pressure concerning a different notion: pointy complexes challenge our normal notions concerning occupation of points. For how the pointy complex occupy the point it is at? Again, there are two main options, corresponding to the ones above. The first is that the point is multiply occupied. That is, the point is occupied by each of the proper parts of the entity: these parts are all exactly located at the point. On this alternative, the parts are co-located. ${ }^{9}$ Or, secondly, the point could be spanned: the whole pointy complex could be located at the point without any of the parts of the entity having locations at all. That is, the point is occupied by no proper part of the entity

\footnotetext{
${ }^{9}$ This option is tacitly assumed in the literature on unextended complexes so far.
} 
but is occupied by the whole entity. On this alternative, the parts have no location. So pointy complexes give us novel ways to think about occupation: either points can be locations for more than one thing or complex entities can have locations without any of their parts having location.

Therefore, pointy complexes are worth considering for those working on theories of location.

(ii) A second application of extended simples is in the context of gunk. To say something is gunky is to say that it has infinitely descending parts: no matter how far down you go you you'll never reach simples. In other words, there are no relevant mereological atoms. Extended simples, by distinguishing the mereological structure of entities and of space, allow us make room for the following possibility: space is gunky but entities are not. So it could be that every part of space itself has (proper) parts, while objects are composed of mereological atoms. These atoms would be extended simples.

As might be anticipated, pointy complexes give us the resources to say the converse: entities are gunky but space is not. It could be that every part of an entity has (proper) parts, while space itself is composed of simple points. The entities occupying these points would be pointy complexes. We can have points without atoms, as well as atoms without points. ${ }^{10}$

(iii) A third place that extended simples have been metaphysically fruitful is as a result of thinking about their properties. While extended simples might seem strange enough, work has been done to try to explain how it is metaphysically possible not only for there to be extended simples, but for them to be heterogeneous. Heterogeneous extended simples are

\footnotetext{
${ }^{10}$ McDaniel (2006) argues for this possibility.
} 
heterogeneous in the sense of exhibiting variation across the space that they occupy. For an example, try to imagine (!) an extended simple rod that occupies some region. Now imagine that this rod is red in one half of the region and green in the other half: it is heterogeneous by displaying variation in its colour across the region it occupies. But of course, being simple, it is not that the rod has a red half and a green half. Rather, the simple entity has variation without parts. This seems problematic, as the simple entity thereby seems both red and green (and these are incompatible).

There has been interesting work on how to make sense of such a scenario. Some alternative proposals include (a) fundamentally distributional properties: properties that grant qualitative features across space and are not reducible to properties that grant qualitative features at points (Parsons), (b) fundamental relativisation of properties to space: properties contain irreducible spatial reference, (c) tropes: properties are tropes particularised to spatial locations (d) distinguishing between things and the stuff of which they are made: things are made of stuff, simple things can be made of complex stuff, and the complex stuff can ground the qualitative variation of the simple things (Markosian). Each of these makes a concrete claim about the metaphysics of properties or entities that applies more generally than just in the case of extended simples. Extended simples are therefore a tool for opening up these metaphysical alternatives.

I want to suggest that pointy complexes offer a similar tool for opening up distinct metaphysical alternatives corresponding to an inversion of those listed above. Suppose that heterogeneous pointy complexes are metaphysically possible. They are heterogeneous in a different sense to extended simples: they exhibit variation across their parts (rather than across space). Heterogeneous pointy complexes, therefore, have qualitative variety in their parts while being spatially homogeneous (because they are only point-sized). For an example of this, first imagine (!) a pointy complex entity that has two parts. Now imagine 
that one of the parts of the entity is red the other part of the entity is green. The entity would thereby display variation across its parts, but of course it is not a certain way at one point and another way at a different point. Instead, the point-sized entity has variation without extension. There are no distinct spaces where the parts of the entity have different properties, but nevertheless the parts differ. The issue then is how to make sense of qualitative variation in parts without variation across space. In other words, how can the parts of a pointy complex be qualitatively different if they are not in different places? How can the point permit the parts to be red and green? More generally, how can a single point of space allow exemplification of seemingly incompatible properties?

A series of corresponding proposals might be considered: ( $\left.a^{\prime}\right)$ fundamentally holistic properties: properties that grant qualitative variation across the parts of an entity and are not reducible to properties of the parts. Although it seems that the parts have distinct properties, in fact there is only a single holistic property had by the whole entity. This means that this single property is the only one exemplified at the point of space the pointy complex occupies, but that the parts exhibit qualitative variation in virtue of this single property, $\left(b^{\prime}\right)$ fundamental relativisation of properties to parts: properties contain irreducible reference to the parts of entities, so that the multiple properties exemplified at the point are in fact compatible (red-with-respect-to-part-1, green-with-respect-to-part-2 etc,), (c') tropes: properties are tropes particularised to parts, so that the point exhibits the part-1-red trope and the part-2-green trope, (d) distinguishing between stuff and the things it makes up: simple stuff can compose complex things, and the complex things can ground the variation of the simple stuff. The parts of the thing are green and red and, in virtue of this, the stuff is too. ${ }^{11}$

\footnotetext{
${ }^{11}$ This option needs the most work. For instance, it might require reversing or at least tinkering with the composition relation. I don't have space to explore this further here.
} 
These four options are rather outlandish ways to conceive of properties, things and stuff. But as far as I can see there's nothing more outlandish here than in the extended simples case. So our views of what properties are and how they can be instantiated in space can be informed by considering pointy complexes.

It is also interesting to reflect on the relationship between solutions to the heterogeneous extended simples and heterogeneous pointy complex cases. If there is a connection between solving the extended simples case and solving the pointy complex case, then considering the latter might give us novel reasons for deciding between competing solutions to the former. But if not, then heterogeneous pointy complexes offer philosophical consequences interestingly distinct from those of heterogeneous extended simples. ${ }^{12}$ In either case, they warrant further investigation.

(iv) Finally, one particular (and extreme) application of extended simples is in a monist picture defended by Schaffer, among others. According to this version of monism, called existence monism, there is really only one thing. This thing is extended. The whole world is therefore an extended simple. Setting aside arguments about this position, we can use unextended complexes to see the correlate view: the whole world is an unextended complex. I will call this view unextensionalism. This will suffer from the opposite worries to the existence monist: rather than trying to explain why what seem to be many entities spread out in space is in fact one entity spread out in space, the proponent of unextensionalism will have to explain why what seem to be many entities spread out in space is in fact many entities not spread out in space. There are very many historical precedents for an unextensionalism view: idealism is a version of it. For the idealist has no (concrete) space, so the whole world will therefore lack extension. But the distinction above between two ways of being

\footnotetext{
${ }^{12}$ I am grateful to a reviewer for flagging this second possibility, and its consequences, to me.
} 
unextended invites consideration of a new version of unextensionalism: the whole world is a pointy complex. On this view, there is (presumably) one and only one point of space, which contains everything concrete. Call this view spatial monism.

I do not offer spatial monism as a plausible candidate for a description of the way the world is. Rather, it is an interesting possibility that is highlighted by considering pointy simples. In much the way that extended simples provides the conceptual and metaphysical gap in which existence monism sits, pointy simples open up a corresponding gap for spatial monism.

\section{Conclusion}

This paper has drawn attention to and examined the notion of unextended complexes, and in particular pointy complexes. I suggest that pointy complexes are no less plausible than extended simples, and that by using them in ways similar to the uses of extended simples we can explore interesting new metaphysical territory. The brief survey in Section 2 is indicative of their potential. Pointy complexes, and unextended simples more generally, should be added to the metaphysician's toolkit. ${ }^{13}$

\section{References}

Armstrong, D. 1988. Are Quantities Relations? A Reply to Bigelow and Pargetter. Philosophical Studies 54: 305-316.

----- 1997. A World of States of Affairs. Cambridge: CUP.

Braddon-Mitchell, D. and Miller, K. 2006. The Physics of Extended Simples. Analysis, 66: 222-226.

\footnotetext{
${ }^{13}$ I am grateful to David Glick and Gonzalo Rodriguez-Pereyra for discussing aspects of this paper with me, and to reviewers for this journal for their helpful and insightful comments.
} 
Eddon, M. 2007. Armstrong on Quantities and Resemblance. Philosophical Studies 136: 385-404

Hudson, H. 2001. A Materialist Metaphysics of the Human Person. Ithca: Cornell University Press.

Markosian, N. 1998. Simples. Australasian Journal of Philosophy, 76: 213-226

2004. Simples, Stuff and Simple People. The Monist, 87: 405-428.

McDaniel, K. 2006. Gunky Objects in a Simple World. Philo, 9: 39-46.

----- 2007a. Extended Simples. Philosophical Studies, 133: 131-141.

----- 2007b. Brutal Simples. Oxford Studies in Metaphysics, vol. 3., 233-266. Ed. Zimmerman. Oxford:

OUP.

---- 2009. Extended Simples and Qualitative Heterogeneity. The Philosophical Quarterly 59: 325331.

Parsons, J. 2000. Must a Four-Dimensionalist Believe in Temporal Parts? The Monist, 83: 399-418.

----- 2007. Theories of Location. Oxford Studies in Metaphysics, vol. 3., 201-232. Ed. Zimmerman.

Oxford: OUP

Saucedo, R. 2011. Parthood and Location. In Oxford Studies in Metaphysics, vol. 6, 225-286.

Ed. Bennett and Zimmerman. Oxford: OUP

Schaffer, J. 2009. Spacetime the one substance. Philosophical Studies, 145: 131-148.

Simons, P. 2004. Extended Simples: A Third Way Between Atoms and Gunk. The Monist, 87: 371384.

Wasserman, R. 2003. Review Article: Hud Hudson's A Materialist Metaphysics of the Human Person. Philo, 6: 307-313. 